\title{
Frequency-domain Subspace Identification of Nonlinear Mechanical Systems - Application to a Solar Array Structure
}

\author{
J.P. Noël ${ }^{1}$, G. Kerschen ${ }^{1}$, E. Foltête ${ }^{2}$, S. Cogan ${ }^{2}$ \\ ${ }^{1}$ Space Structures and Systems Laboratory (S3L) \\ Department of Aerospace and Mechanical Engineering \\ University of Liège, Liège, Belgium \\ jp.noel, g.kerschen@ulg.ac.be \\ ${ }^{2}$ FEMTO-ST Institute \\ LMARC, Besançon, France \\ emmanuel.foltete,scott.cogan@univ-fcomte.fr
}

\begin{abstract}
The present paper addresses the experimental identification of a simplified realisation of a solar array structure in folded configuration. To this end, a nonlinear subspace identification technique formulated in the frequency domain, referred to as the FNSI method, is exploited. The frequency response functions of the underlying linear structure and the nonlinear coefficients are estimated by this approach. Nonlinearity is caused by impacts between adjacent panels and friction and gaps appearing in their clamping interfaces. This application is challenging for several reasons, which include high modal density and the complicated nature of the involved nonlinear mechanisms.
\end{abstract}

Keywords: Nonlinear system identification; subspace method; frequency domain; space structure; experimental data.

\section{INTRODUCTION}

Identification of linear and time-invariant systems is a discipline that has evolved considerably during the last forty years. The techniques available today are really quite sophisticated and advanced. In particular, subspace algorithms ${ }^{[1,2]}$ are powerful identification methods that are routinely used for experimental and operational modal analysis ${ }^{[3]}$, but also for advanced processing such as damage detection and structural health monitoring ${ }^{[4]}$. However, nonlinearity is a frequent occurrence in engineering structures and, for this reason, subspace-based methods have recently been generalised to handle nonlinear systems. In particular, the frequency-domain nonlinear subspace identification (FNSI) method has proved successful ${ }^{[5,6]}$.

Space structure dynamics is a typical field of application for nonlinear system identification techniques ${ }^{[7]}$. Indeed, although they are generally modelled as linear systems, space structures are known to be prone to nonlinear phenomena. A specific difficulty encountered is the behaviour of solar generators in their stowed position. In fact, solar panels are folded during the spacecraft launch to save volume and impact one another at specific points, leading to nonlinear boundary conditions. As the excitation level increases, friction and gaps may also appear in the clamping interfaces between adjacent panels. In this context, the objective of the present paper is to address the experimental identification of a simplified realisation of a solar array structure in folded configuration using the FNSI method. The frequency response functions (FRFs) of the underlying linear structure and the nonlinear coefficients will be estimated by this approach. This application is challenging for several reasons, which include high modal density and the complicated nature of the involved nonlinear mechanisms. The paper is organised as follows. Section 2 
describes the experimental test rig and its instrumentation. The FNSI method is then introduced in Section 3 and applied to the solar array structure using random and sine-sweep data in Section 4. The conclusions of this application are finally summarised in Section 5.

\section{DESCRIPTION OF A SIMPLIFIED SOLAR ARRAY SYSTEM}

In response to the issue raised in the introductory section, a simplified experimental test rig that mimics the complex dynamics of folded solar generators has been built. It consists of two $77 \times 44 \times 0.5 \mathrm{~cm}^{3}$ parallel aluminium plates clamped together on one edge and connected through three stacking points, as pictured in Figure 1 (a). The system is mounted in free-free configuration. Imperfect tightening of bolts within the stacking points are suspected to generate friction and clearance. In addition, two solithane snubbers mounted on steel supports are introduced on both corners of the free edge (Figure 1 (b)). They are designed so as to maintain contact and absorb vibrations. At high excitation level however, solithane material nonlinearity may emerge and the loss of contact between adjacent panels may also result in impacts ${ }^{[8]}$. In this study, a freeplay of less than a millimetre is introduced such that there is no contact at rest (see Figure 1 (c)). This allows the proper definition of a linear regime of motion at low amplitude.

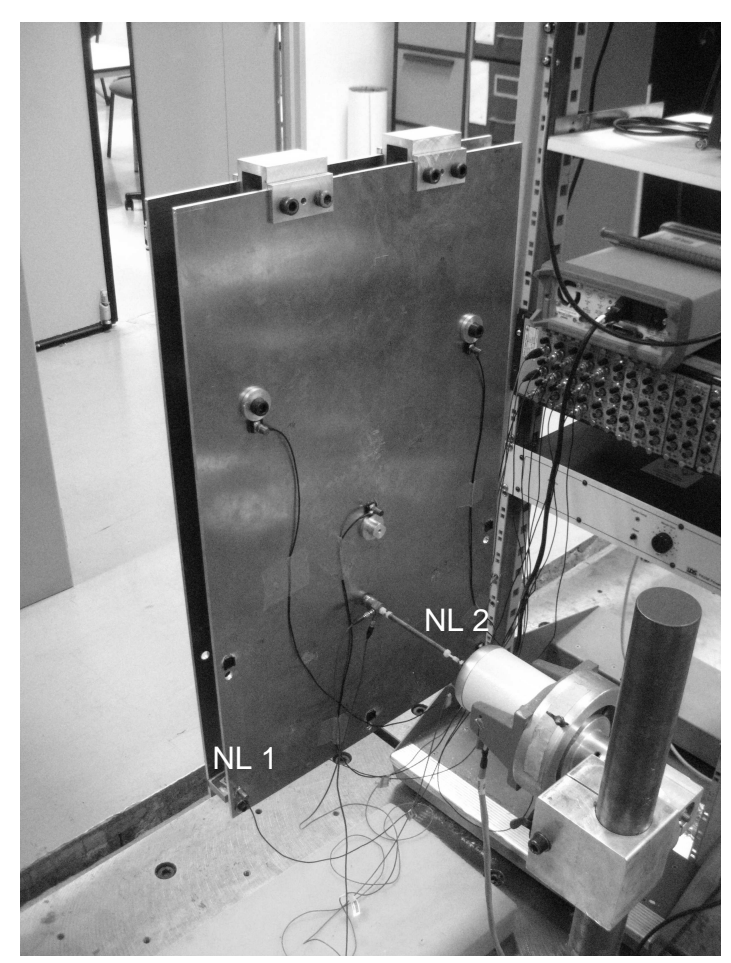

(a)

(b)

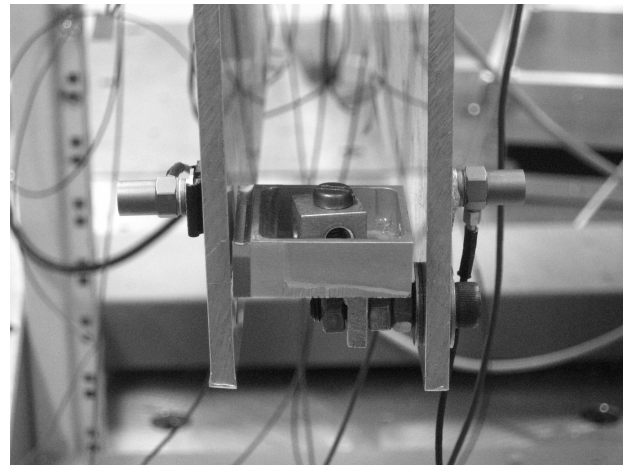

(c)

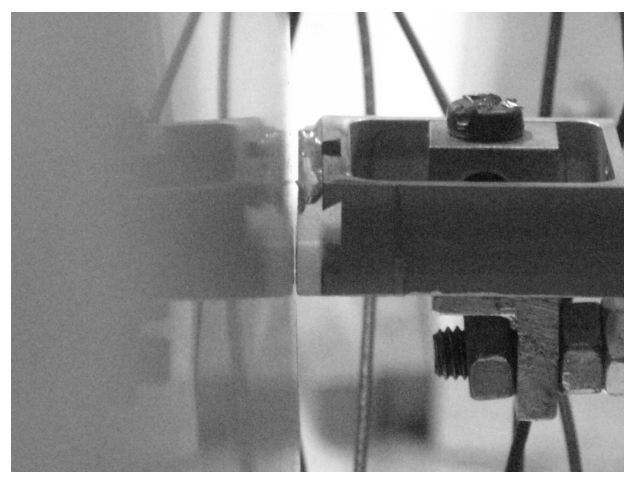

Figure 1: Simplified solar array system. (a) Global view of the two aluminium plates clamped together at the top edge; (b) close-up on the NL 1 snubber connection instrumented with one accelerometer at both ends; (c) very thin freeplay between the snubber and the rear panel in NL 2.

The structure is instrumented with 10 accelerometers positioned at both ends of each suspected nonlinearity, i.e. the 3 stacking points and the 2 snubbers (Figure 1 (b)). Besides, a 10-Newton shaker, visible in Figure 1 (a), is used to apply random and sinesweep excitations, considering a sampling frequency of $6,400 \mathrm{~Hz}$. Acceleration and force signals are recorded at the excitation point, located below the stacking points, through an impedance head. For the sake of conciseness, the present paper will exclusively focus on the identification of the two snubber connections, labelled NL 1 and NL 2, which will be shown to exhibit a combined softening-hardening behaviour. 


\section{SUBSPACE IDENTIFICATION OF NONLINEAR MECHANICAL SYSTEMS IN THE FREQUENCY DOMAIN}

One way of tackling nonlinear system identification in structural dynamics is to break the problem down into three successive tasks, namely detection, characterisation and parameter estimation ${ }^{[9]}$. Following nonlinearity detection whose goal is obvious, characterisation is concerned with nonlinearity location and model selection. The model parameters then introduced are generally estimated through least-squares fitting, or nonlinear optimisation. The FNSI method addresses this latter step and so aims at estimating nonlinear stiffness and/or damping coefficients and the FRF matrix of the underlying linear system.

The vibrations of nonlinear systems which possess an underlying linear regime of motion are governed by the time-continuous model

$$
M \ddot{q}(t)+C \dot{q}(t)+K q(t)+f(q(t), \dot{q}(t))=p(t)
$$

where $M, C, K \in \mathbb{R}^{r \times r}$ are the linear mass, damping and stiffness matrices, respectively; $q(t)$ and $p(t) \in \mathbb{R}^{r}$ are the generalised displacement and external force vectors, respectively; $f(t) \in \mathbb{R}^{r}$ is the nonlinear restoring force vector, and $r$ is the number of degrees of freedom (DOFs) of the structure obtained after spatial discretisation. The amplitude, the direction, the location and the frequency content of the excitation $p(t)$ determine in which regime the structure behaves. As in reference ${ }^{[10]}$, the effects of the $s$ lumped nonlinearities are modelled using the summation

$$
f(q(t), \dot{q}(t))=\sum_{j=1}^{s} \mu_{j} b_{j} g_{j}(q(t), \dot{q}(t)) .
$$

Each term contains an unknown nonlinear coefficient $\mu_{j}$ and the corresponding functional form $g_{j}(t)$, which is assumed to be known. Nonlinearity location is specified through a vector of boolean values, $b_{j} \in \mathbb{R}^{r}$. In the literature about subspace methods, first-order state-space models are universally preferred to the second-order description of the dynamics in Equation (1), because the intrinsic capability of a state-space model to encompass multi-input multi-output systems is attractive. Assuming that displacements are measured and defining the state vector $x=\left(\begin{array}{ll}q^{T} & \dot{q}^{T}\end{array}\right)^{T} \in \mathbb{R}^{n}$, the equations of motion are recast into

$$
\left\{\begin{array}{l}
\dot{x}(t)=A_{c} x(t)+B_{c}^{n l} g(t)+B_{c} p(t) \\
q(t)=C_{c} x(t)+D_{c} p(t)
\end{array}\right.
$$

where subscript $c$ stands for continuous-time; $A_{c} \in \mathbb{R}^{n \times n}, B_{c}^{n l} \in \mathbb{R}^{n \times s}, B_{c} \in \mathbb{R}^{n \times r}, C_{c} \in \mathbb{R}^{r \times n}$ and $D_{c} \in \mathbb{R}^{r \times r}$ are the state, nonlinear coefficient, input, output and direct feedthrough matrices, respectively; $g(t) \in \mathbb{R}^{s}$ gathers the basis functions $g_{j}(t)$, and $n=2 r$. State-space and physical-space matrices correspond through the relations

$$
\begin{gathered}
A_{c}=\left(\begin{array}{cc}
0^{r \times r} & \mathrm{I}^{r \times r} \\
-M^{-1} K & -M^{-1} C
\end{array}\right) \quad B_{c}^{n l}=\left(\begin{array}{cccc}
0^{r \times 1} & 0^{r \times 1} & \cdots & 0^{r \times 1} \\
-\mu_{1} M^{-1} b_{1} & -\mu_{2} M^{-1} b_{2} & \ldots & -\mu_{s} M^{-1} b_{s}
\end{array}\right) \\
B_{c}=\left(\begin{array}{c}
0^{r \times r} \\
M^{-1}
\end{array}\right) \quad C_{c}=\left(\begin{array}{lll}
l^{r \times r} & 0^{r \times r}
\end{array}\right) \quad D_{c}=0^{r \times r}
\end{gathered}
$$

where 0 and I are zero and identity matrices, respectively. Given $p(t)$ and $q(t)$, the FNSI method determines the five matrices $A_{c}, B_{c}^{n l}, B_{c}, C_{c}$ and $D_{c}$. The estimation of the nonlinear coefficients $\mu_{j}$ and of the FRFs is subsequently carried out thanks to the conversion from state space to physical space outlined in Section 3.4.

\subsection{Equivalent linear identification through feedback}

The FNSI method utilises the feedback formulation proposed in ${ }^{[10]}$, and illustrated in Figure 2, for interpreting the dynamics governed by Equation (1). It consists in moving the nonlinear term to the right-hand side of this equation and viewing nonlinear forces as external forces applied to the underlying linear structure. Thus, the internal forces that are nonlinear functions of the outputs act as a feedback to the linear open-loop system. Considering Equations (3), this interpretation boils down to the concatenation of $g(t)$ and $p(t)$ into a single extended input vector $e(t) \in \mathbb{R}^{s+r}$ :

$$
\left\{\begin{aligned}
\dot{x}(t) & =A_{c} x(t)+B_{c}^{e} e(q(t), \dot{q}(t)) \\
q(t) & =C_{c} x(t)+D_{c}^{e} e(t)
\end{aligned}\right.
$$

where $B_{c}^{e}=\left(\begin{array}{ll}B_{c}^{n l} & B_{c}\end{array}\right) \in \mathbb{R}^{n \times(s+r)}$ and $D_{c}^{e}=\left(\begin{array}{ll}0^{r \times s} & D_{c}\end{array}\right) \in \mathbb{R}^{r \times(s+r)}$. The feedback formulation is particularly appealing, because the inverse problem to be solved is now equivalent to the widely-studied linear state-space identification problem. 
However, because the FNSI algorithm handles nonlinearities in input-output data, its interpretation and use must be tailored, as detailed in reference ${ }^{[5]}$.

As extensively discussed by Pintelon and Schoukens in ${ }^{[11]}$, tackling system identification problems in the frequency domain is an attractive and versatile alternative. For improved numerical conditioning ${ }^{[12]}$, a discrete-time translation of Equations (5) is first considered, before applying the discrete Fourier transform (DFT). Provided that the time signal $v(t)$ is periodic and observed over an integer number of periods in steady-state conditions, its DFT $V(k)$ is given by

$$
V(k)=\frac{1}{\sqrt{M}} \sum_{t=0}^{M-1} v(t) e^{-j 2 \pi k t / M}
$$

where $M$ is the number of recorded time samples, $k$ is the frequency line and $j$ the imaginary unit. Equations (5) eventually write

$$
\left\{\begin{aligned}
z_{k} X(k) & =A_{d} X(k)+B_{d}^{e} E(k) \\
Q(k) & =C_{d} X(k)+D_{d}^{e} E(k)
\end{aligned}\right.
$$

where subscript $d$ stands for discrete-time; $z_{k}=e^{j 2 \pi k / M}$ is the Z-transform variable, and $X(k), E(k)$ and $Y(k)$ are the DFTs of $x(t), e(t)$ and $y(t)$, respectively.

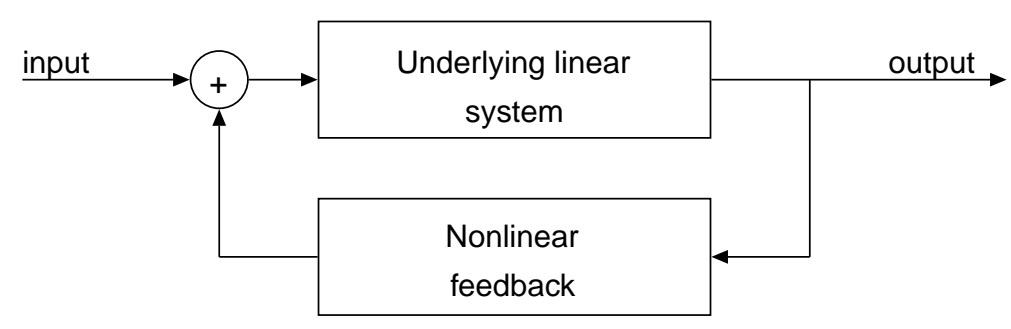

Figure 2: Feedback interpretation of nonlinear mechanical systems ${ }^{[10]}$.

\subsection{Output-state-input matrix equation formulation}

In practice, only a limited set of DOFs in $p(t)$ and $q(t)$ are excited and observed, respectively. The identification problem is therefore preferably stated in terms of the measured applied forces $u(t) \in \mathbb{R}^{m \leq r}$ and displacements $y(t) \in \mathbb{R}^{l \leq r}$. Accordingly, the extended input vector is $e(t) \in \mathbb{R}^{\sigma=s+m}$. Equations (7) become

$$
\left\{\begin{aligned}
z_{k} X(k) & =A_{d} X(k)+B_{d}^{e} E(k) \\
Y(k) & =C_{d} X(k)+D_{d}^{e} E(k)
\end{aligned}\right.
$$

where $Y(k)$ is the DFT of $y(t)$ and the matrices $A_{d}, B_{d}^{e}, C_{d}$ and $D_{d}^{e}$ are now a projection of the original matrices onto the observed and controlled DOFs. In what follows, subscript $d$ indicating discrete-time matrices will be skipped, because no ambiguity is possible.

The measured output frequency spectra matrix is defined as

$$
Y_{i}=\left(\begin{array}{cccc}
Y(1) & Y(2) & \ldots & Y(N) \\
z_{1} Y(1) & z_{2} Y(2) & \ldots & z_{N} Y(N) \\
z_{1}^{2} Y(1) & z_{2}^{2} Y(2) & \ldots & z_{N}^{2} Y(N) \\
\vdots & & & \\
z_{1}^{i-1} Y(1) & z_{2}^{i-1} Y(2) & \ldots & z_{N}^{i-1} Y(N)
\end{array}\right) \in \mathbb{R}^{l i \times N}
$$

where $i$ is a user-defined index and $N$ the number of (non-necessarily equidistant) frequency lines exploited in the identification. Defining $\zeta=\operatorname{diag}\left(z_{1} z_{2} \ldots z_{N}\right) \in \mathbb{R}^{N \times N}, Y_{i}$ is recast into

$$
Y_{i}=\left(\begin{array}{ccccc}
Y^{T} & Y \zeta^{T} & Y \zeta^{2^{T}} & \ldots & Y \zeta^{i-1^{T}}
\end{array}\right)^{T}
$$


The extended input frequency spectra matrix is similarly formed as

$$
E_{i}=\left(\begin{array}{lllll}
E^{T} & E \zeta^{T} & E \zeta^{2 T} & \ldots & E \zeta^{i-1 T}
\end{array}\right)^{T} \in \mathbb{R}^{\sigma i \times N} .
$$

Introducing the extended observability matrix

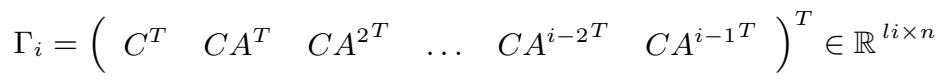

and the lower-block triangular Toeplitz matrix

$$
H_{i}=\left(\begin{array}{ccccc}
D^{e} & 0 & 0 & \ldots & 0 \\
C B^{e} & D^{e} & 0 & \ldots & 0 \\
C A B^{e} & C B^{e} & D^{e} & \ldots & 0 \\
\vdots & \vdots & \vdots & & \vdots \\
C A^{i-2} B^{e} & C A^{i-3} B^{e} & C A^{i-4} B^{e} & \ldots & D^{e}
\end{array}\right) \in \mathbb{R}^{l i \times \sigma i},
$$

recursive substitution of Equations (8) results in the output-state-input matrix equation

$$
Y_{i}=\Gamma_{i} X+H_{i} E_{i}
$$

where $X \in \mathbb{R}^{n \times N}$ is the state spectrum.

Remark that $i$ and $N$ must be chosen to encompass sufficient valuable information to identify the system. The choice of $N$ is discussed in Section 4 where the possibility to focus on frequency regions of interest will prove to be a major asset of the FNSI method. A physics-based or information-based decision about $i$ is more delicate. Basically, the larger $i$, the more accurate the identification, since $i$ conveys how system dynamics is included in the data matrices. However, redundant information can affect the conditioning of those matrices, hence imposing bounds to $i$. There also exists an obvious trade-off between the values of $i$ and $N$ and the time needed to inverse the model.

\subsection{Estimation of the state matrices}

The FNSI algorithm is a three-step procedure built upon Equation (14). First, an estimate of the extended observability matrix $\Gamma_{i}$ is computed. To this end, the term depending on the input and the nonlinearities in Equation (14), namely $H_{i} E_{i}$, is eliminated using a geometrical projection. The vector interpretation of Equation (14) depicted in Figure 3 shows that an orthogonal projection onto the orthogonal complement of $E_{i}$, denoted $E_{i}^{\perp}$, cancels the input term.

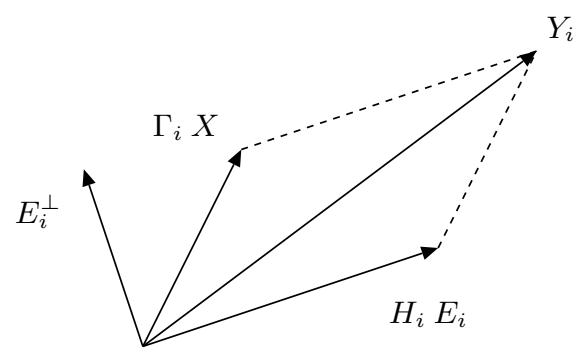

Figure 3: Geometrical interpretation of Equation (14) in a two-dimensional space.

Matrix $\Gamma_{i}$ can then be obtained through a truncated singular value decomposition (SVD) of the result of the projection. The truncation limits the singular value spectrum to genuine elements, hence removing spurious values and reducing the influence of noise and rounding errors on the identification. In addition, the number of retained singular values yields the system order $n$. From the knowledge of $n$ and $\Gamma_{i}$, the second step consists in computing the matrices $A$ and $C$ thanks to the shift property $\underline{\Gamma_{i}} A=\overline{\Gamma_{i}}$, where $\underline{\Gamma_{i}}$ and $\overline{\Gamma_{i}}$ are the matrix $\Gamma_{i}$ without the last and first $l$ rows (or block row), respectively. State matrix $A$ is thus 
found as the least-squares solution of the overdetermined system of equations $A=\Gamma_{i}^{\dagger} \overline{\Gamma_{i}}$, where $\dagger$ is the pseudo-inverse; output matrix $C$ is extracted from $\Gamma_{i}$ as its $l$ first rows. The final step is the estimation of the system matrices $B^{e}$ and $D^{e}$. A robust numerical scheme is described in reference ${ }^{[5]}$. It exploits the formulation of a set of linear equations in $B^{e}$ and $D^{e}$, explicitly solved in least-squares sense. For the sake of conciseness, it is not detailed herein.

\subsection{Conversion from state space to physical space}

Starting from the identified spate-space model $\left(A, B^{e}, C, D^{e}\right)$, the estimation of the nonlinear coefficients $\mu_{j}$ and of the FRF matrix of the underlying linear system $H(\omega)$ can be carried out. To that purpose, a transformation back to physical space is achieved through the derivation of a linear relationship between output and extended input spectra in lumped nonlinear structures, extending the concept of FRF ${ }^{[10]}$ :

$$
Y(\omega)=H(\omega)\left[I^{r \times r}-\sum_{j=1}^{s} \mu_{j} b_{j}\right] E(\omega)=H^{e}(\omega) E(\omega)
$$

where the linear operator $H^{e}(\omega)$ is the extended FRF matrix. Moreover, in ${ }^{[13]}$, the authors proved that $H^{e}(\omega)$ is an invariant system property. It can be retrieved, as in linear theory, from the combination of the continuous-time state-space matrices

$$
H^{e}(\omega)=C_{c}\left(\left.j \omega\right|^{n \times n}-A_{c}\right)^{-1} B_{c}^{e}+D_{c}^{e}
$$

As a result, the nonlinear coefficients identified from the extended FRF matrix are spectral quantities, i.e. they are complex-valued and frequency-dependent. A reliable identification scheme together with an appropriate selection of the nonlinear functional forms should make the imaginary parts much smaller than the corresponding real parts. The frequency dependence of the coefficients should also remain small. These indications will serve as quality criteria in Section 4.

\section{IDENTIFICATION OF THE SNUBBER SOFTENING-HARDENING NONLINEARITIES}

Since the choice was made to introduce a static gap between the two snubbers and the rear panel, a linear analysis of the structure can safely be achieved at low level. To this end, a band-limited $(5-350 \mathrm{~Hz})$ white-noise signal was applied to the structure during 90 seconds considering a root-mean-square (RMS) amplitude of $0.16 \mathrm{~N}$. Figure 4 shows the linear properties extracted by the FNSI method for model orders up to 200. The number of block rows $i$ was chosen equal to 100 and measured frequency samples were processed in the excitation band only. This stabilisation diagram leads to the identification of 21 modes, whose natural frequencies and damping ratios are listed in Table 1. As expected for an aluminium structure, damping ratios are all very low and are bounded above by $1 \%$. The FRF superimposed in Figure 4 was measured on the front panel in NL 1, and shows an excellent agreement with the estimated linear frequencies. Finally, the deformed shape of mode 10 at $190.60 \mathrm{~Hz}$ is depicted in Figure 5 and corresponds to an out-of-phase motion of the two panels. This mode will be found to be of prime importance for nonlinear identification purposes since it is likely to involve impacts for increasing excitation levels.

A second random data set measured at high level (3.82 NRMS) is now analysed to detect and locate in the frequency domain the modes triggering nonlinear mechanisms. Figure 6 (a) presents the comparison between the low- and high-level FRFs measured on the front panel in NL 1. One first observes that some peaks are visibly not affected by nonlinearity, e.g. around 59, 62 or 145 $\mathrm{Hz}$. Others clearly suffer from a shift towards lower frequencies, as a symptom of a softening behaviour, e.g. around 241,264 or $339 \mathrm{~Hz}$. This can arguably be attributed to loosening of bolts within the stacking points but will not be analysed further in the present work for brevity purposes. Eventually, three modes around 32, 46 and $191 \mathrm{~Hz}$ exhibit an increase of their resonance frequency and a "noisy" behaviour typical of hardening nonlinearities. They most probably translate the presence of impacts in the measurements. The variety of nonlinear distortions involved in the system response is well illustrated in the frequency close-up provided in Figure 6 (b) where three successive modes are found to manifest linear, softening and hardening behaviour, respectively. In the sequel, we will focus on the identification of the nonlinearities induced by the presence of snubbers, and will further restrict our interest to the mode at $190.60 \mathrm{~Hz}$ (see Figure 5) which involves the largest relative displacements between the two panels and hence the most severe impacts.

Following nonlinearity detection, the adequate selection of the describing functions $g_{j}(t)$ is a key step to the success of the estimation of nonlinear coefficients. In this context, the restoring force surface (RFS) method is worth being utilised since it yields a convenient visualisation of nonlinear effects. The RFS method relies on Newton's second law of motion, written for a single-degree-of-freedom system as

$$
m \ddot{x}+f(x, \dot{x})=p
$$




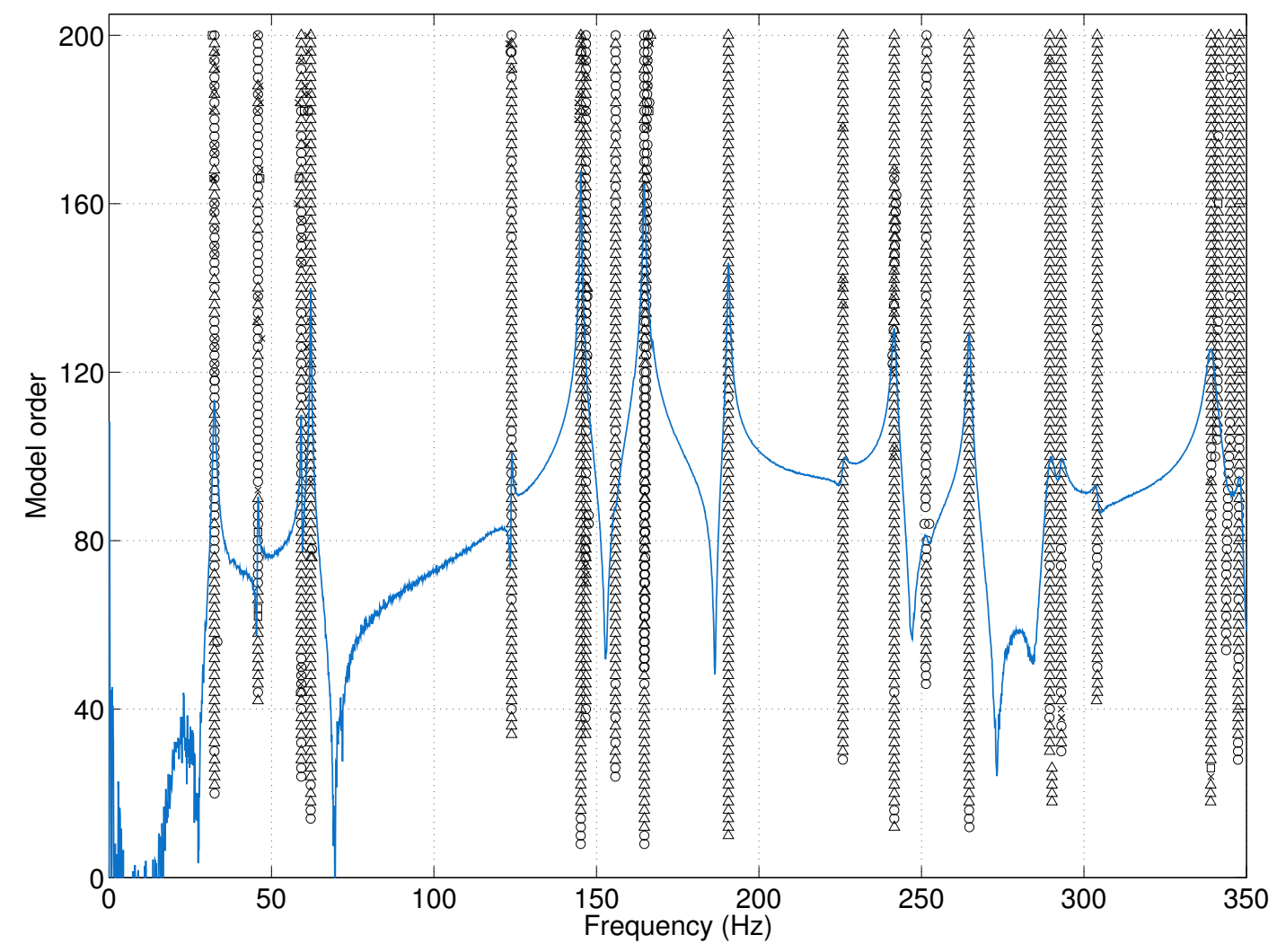

Figure 4: Stabilisation diagram computed by the FNSI method at low level and corresponding FRF measured in NL 1 on the front panel. A cross denotes a pole stabilised in frequency only. Extra-stabilisation in damping and mode shape are given by squares and circles, respectively. Full stabilisation is expressed through a triangle. Stabilisation thresholds in natural frequency, damping ratio and modal assurance criterion (MAC) value were set to $0.1 \%, 1 \%$ and 0.99 , respectively.

where $m$ is the mass, $\ddot{x}$ the acceleration and $p$ the external force and where $f$ encompasses all the restoring forces in the system, being of elastic or dissipative nature. This equation recast into

$$
f(x, \dot{x})=p-m \ddot{x}
$$

gives a direct access to a nonparametric estimate of the restoring force surface defined by the triplets $(x, \dot{x}, f(x, \dot{x}))$. Applied to more complex systems, the method only provides qualitative information but can still be exploited for nonlinearity characterisation. Figures $7(a, b)$ depict the stiffness curves computed across the NL 1 and NL 2 connections, respectively. As the use of the RFS method is known to be facilitated when the modes can be isolated in the structural response, a 4-Newton sine-sweep excitation is here considered. Both curves present a large increase of stiffness in negative relative displacements which is the clear indication of impacts between the snubbers of the rear panel. The clearances beyond which contact occurs can be estimated at 0.08 and $0.1 \mathrm{~mm}$ in NL 1 and NL 2, respectively. A global softening of stiffness in the system is also observed in Figure 7 and is probably due to bolt loosening in the stacking points. This latter effect will be modelled as a negative cubic describing function.

The FNSI method can eventually be applied to the 3.82 NRMS random data set considering four nonlinearities as input forces, i.e. two bilinear and two negative cubic forces. For instance, Figure 8 shows the describing function chosen in NL 2, consisting of a bilinear stiffness curve whose discontinuity is located in $-0.1 \mathrm{~mm}$ superimposed to a negative cubic stiffness curve. The real parts of the four identified nonlinear coefficients $\mu_{j}$ are displayed in Figure 9 and are found to be stable against frequency, as expected from good-quality estimates. Note that measured frequency samples were exclusively processed in the interval 185 $-200 \mathrm{~Hz}$. This demonstrates one of the major assets of the FNSI method, namely its capability to discriminate frequencies in terms of information content. In particular, the focus of the analysis on the neighbourhood of the $190.60 \mathrm{~Hz}$ mode would merely be impossible in the time domain, thus considerably complicating the identification.

The averaged values of the coefficients are given in Table 2 and a good agreement is observed between the two nonlinear 


\begin{tabular}{ccc} 
Mode & Frequency $(\mathrm{Hz})$ & Damping ratio $(\%)$ \\
\hline & 32.47 & 0.81 \\
2 & 45.85 & 0.44 \\
3 & 59.17 & 0.18 \\
4 & 62.08 & 0.17 \\
5 & 123.88 & 0.14 \\
6 & 145.18 & 0.10 \\
7 & 146.74 & 0.25 \\
8 & 155.85 & 0.37 \\
9 & 164.73 & 0.17 \\
10 & 190.60 & 0.14 \\
11 & 225.84 & 0.35 \\
12 & 241.62 & 0.24 \\
13 & 251.40 & 0.34 \\
14 & 264.66 & 0.17 \\
15 & 289.48 & 0.48 \\
16 & 292.95 & 0.31 \\
17 & 304.10 & 0.27 \\
18 & 339.16 & 0.38 \\
19 & 341.24 & 0.55 \\
20 & 344.97 & 0.19 \\
21 & 347.80 & 0.24
\end{tabular}

TABLE 1: Identified frequencies and damping ratios in $5-350 \mathrm{~Hz}$ using a FNSI stabilisation diagram.

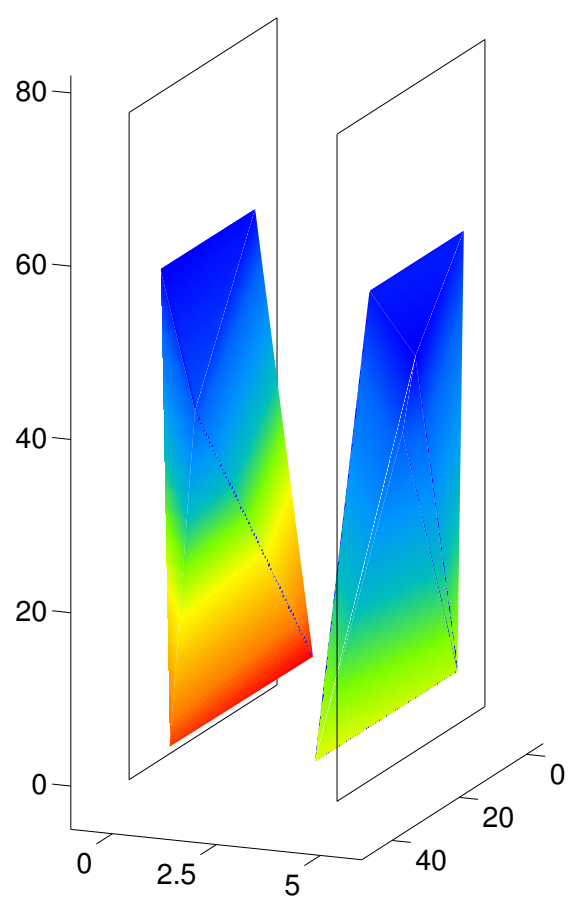

Figure 5: Deformed shape of mode 10 at $190.60 \mathrm{~Hz}$. Geometrical dimensions are given in centimetres.

connections. Table 2 also lists the ratios, in logarithmic scale, between the real and imaginary parts of the coefficients. They are found to be meaningful quality indicators since the more stable a coefficient, the larger the corresponding ratio. Besides, the FNSI method can be exploited to reconstruct the FRFs of the underlying linear system. Figure 10 shows the improvement brought by the FNSI estimate with respect to the result of a classical linear estimator. The associated natural frequency closely matches the low-level property with a relative error of $0.04 \%$. The damping ratio is also satisfactorily estimated at $0.21 \%$, though suffering from a larger relative error $(51 \%)$.

\section{CONCLUSIONS}

This paper intended to carry out the identification of simplified solar array structure based on experimental data. To this end, a frequency-domain nonlinear generalisation of subspace methods, referred to as the FNSI method, was exploited. The focus was put onto a single mode of vibration involving both impact and material nonlinearities. The corresponding nonlinear stiffness coefficients were estimated, and the FNSI method was also shown to accurately reconstruct the FRFs of the underlying linear structure. However, additional investigations are needed to further understand the dynamics of the structure so as to improve the predictive capabilities of an identified model. In particular, attention should now be devoted to the study of bolt loosening within the stacking points.

\section{ACKNOWLEDGEMENTS}

The author J.P. Noël would like to acknowledge the Belgian National Fund for Scientific Research (FRIA fellowship) for its financial support. 


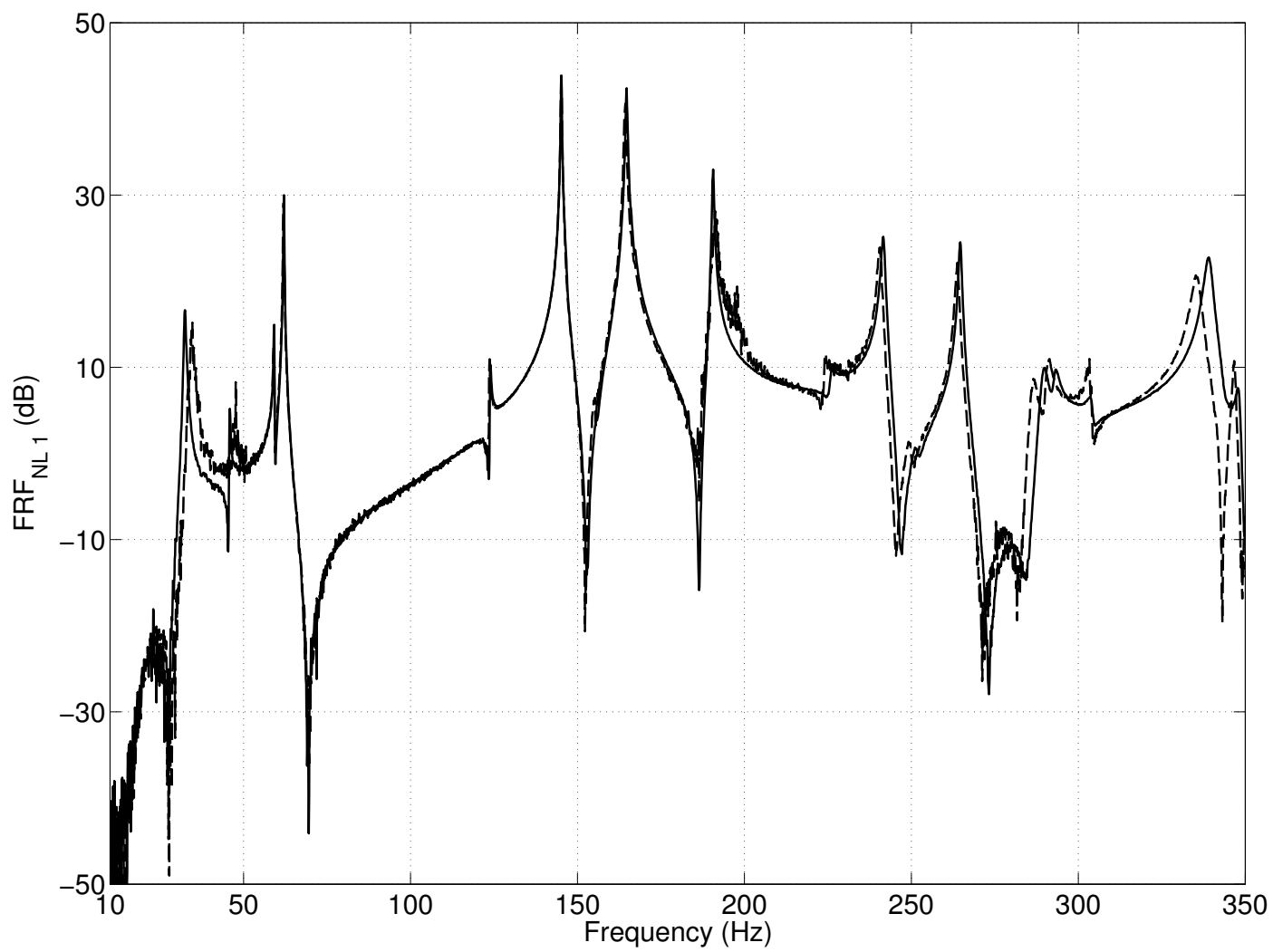

(a)

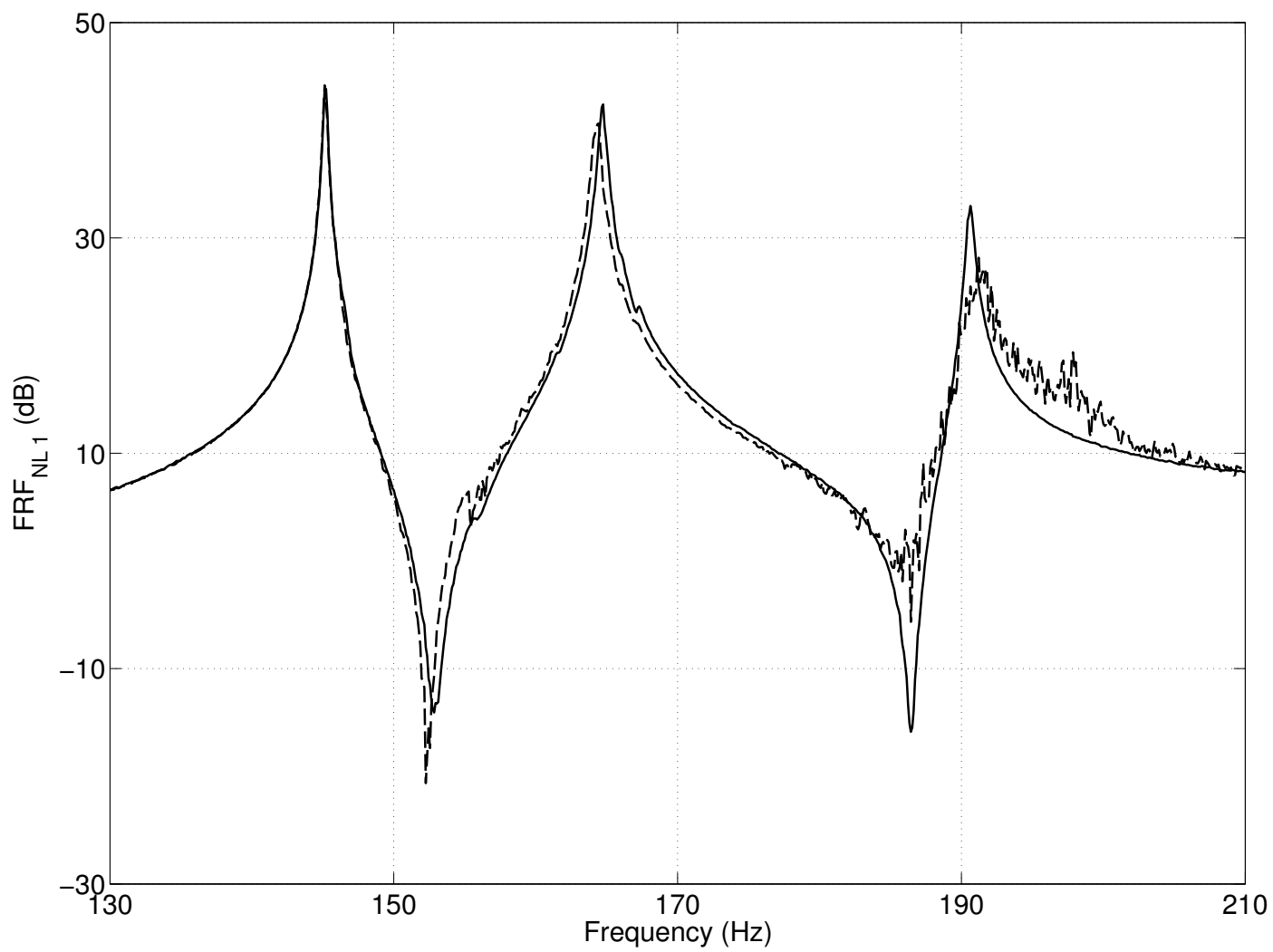

(b)

Figure 6: Detection of nonlinearity in the frequency domain using FRF plots measured in NL 1 on the front panel. Solid line: 0.16 NRMS; dashed line: 3.82 NRMS. (a) Full excitation band; (b) close-up on three modes exhibiting different nonlinear behaviours. 


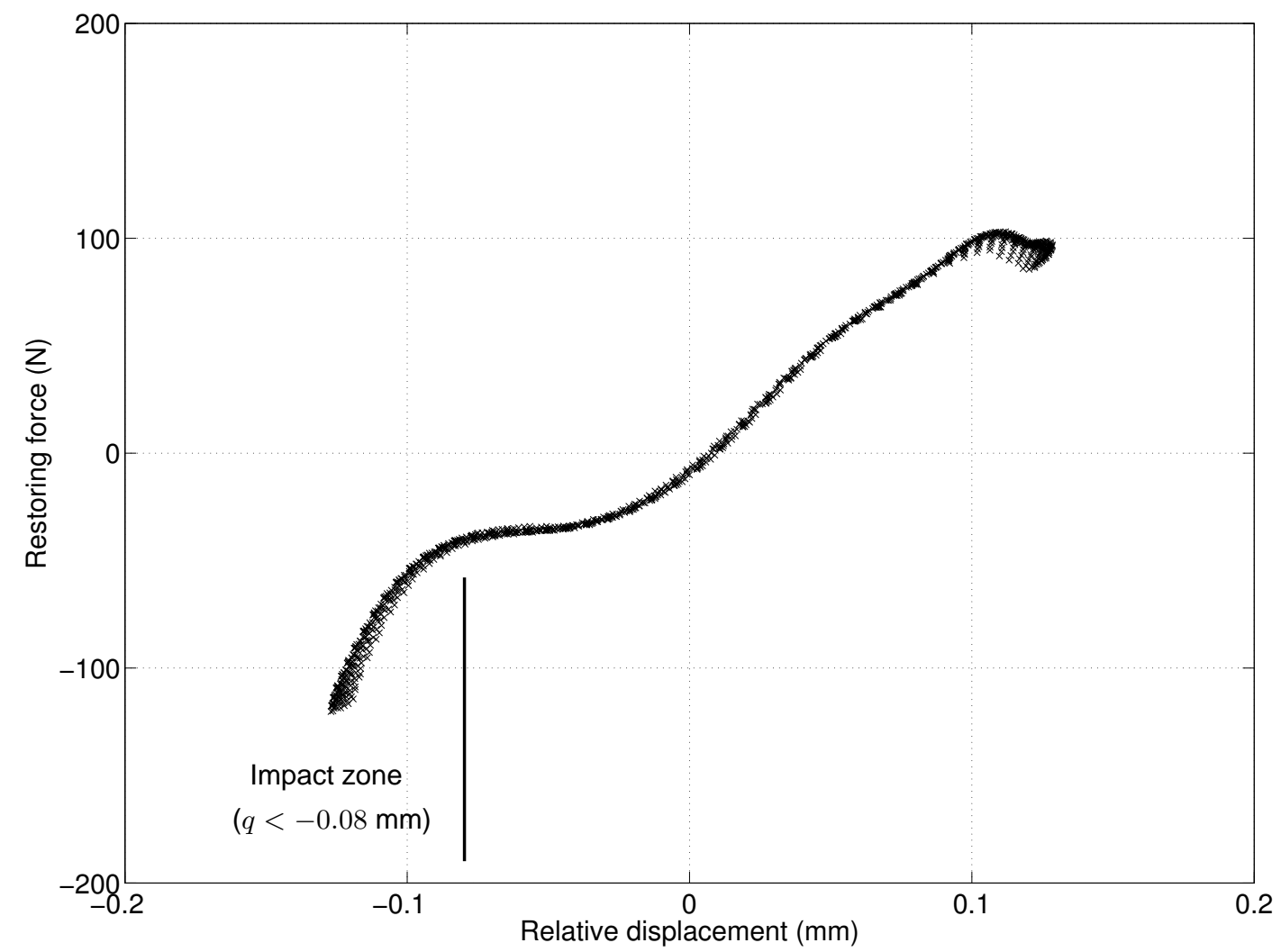

(a)

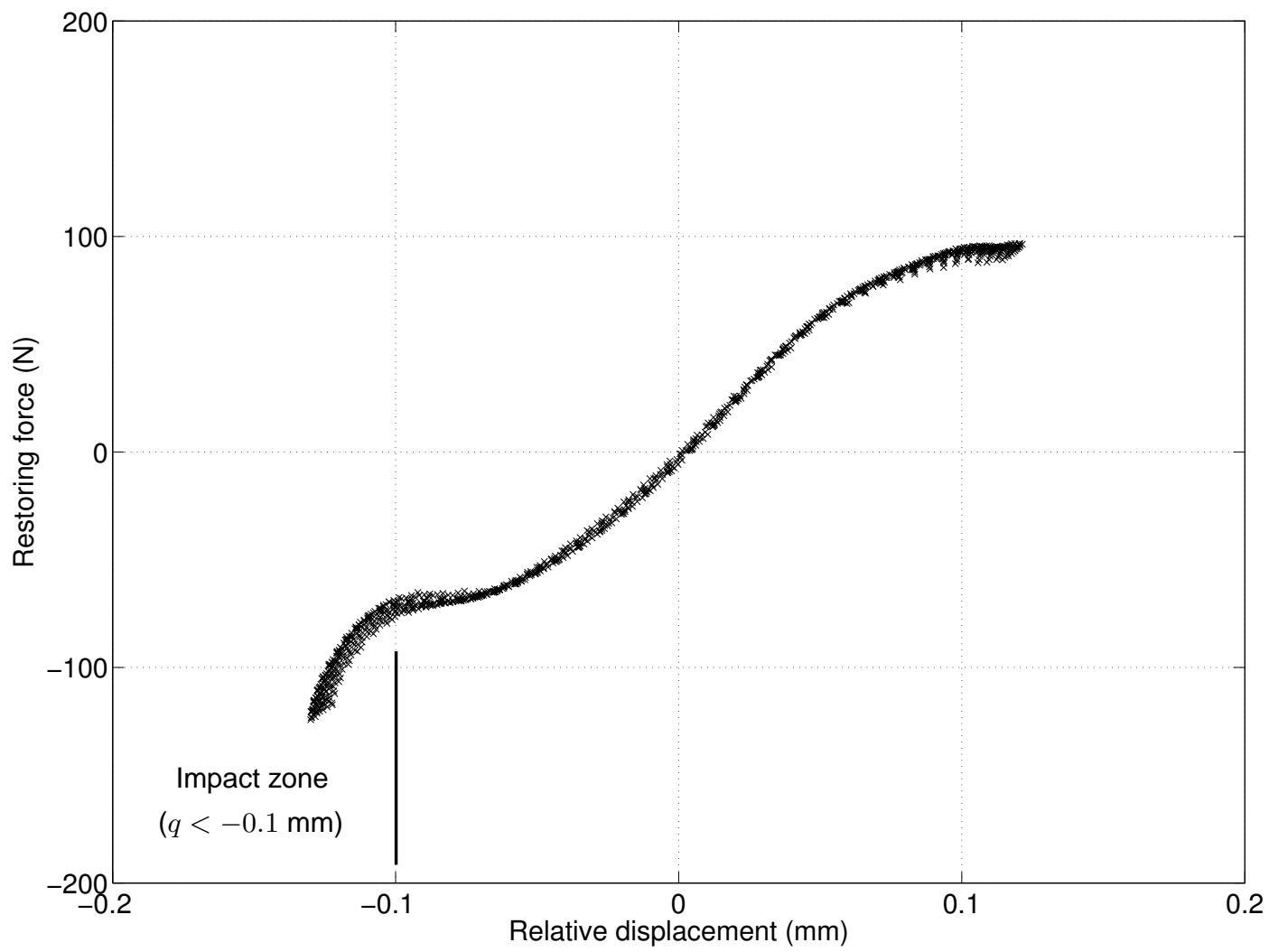

(b)

Figure 7: RFS-based stiffness curves computed in the neighbourhood of mode $10(190-191 \mathrm{~Hz})$. The excitation is a 4-Newton sine signal sweeping the $5-350 \mathrm{~Hz}$ band at $172.5 \mathrm{~Hz} / \mathrm{min}$. (a) NL 1; (b) NL 2 . 


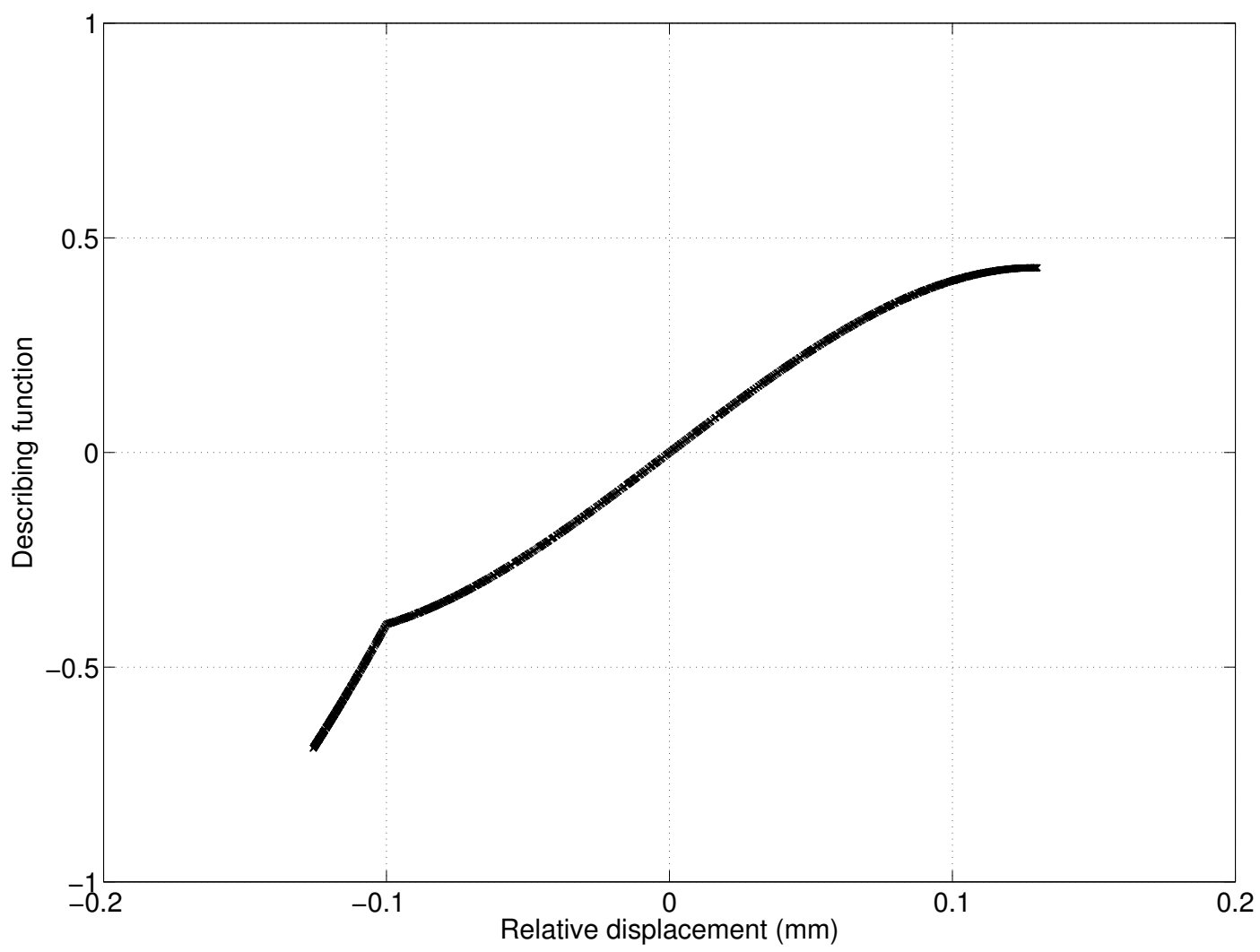

Figure 8: Describing function chosen to model the nonlinearity observed in NL 2 in Figure 7 (b). It consists of a bilinear function whose discontinuity is located in $-0.1 \mathrm{~mm}$ superimposed to a negative cubic function.

\begin{tabular}{cccc}
\hline Nonlinear connection & Describing function & Real part & $\log _{10}$ (Real//mag.) \\
NL 1 & $q<-0.08 \mathrm{~mm}$ & $5.6 \mathrm{kN} / \mathrm{m}$ & 0.46 \\
& $-q^{3}$ & $319 \mathrm{MN} / \mathrm{m}^{3}$ & 0.67 \\
NL 2 & $q<-0.1 \mathrm{~mm}$ & $12.8 \mathrm{kN} / \mathrm{m}$ & 0.43 \\
& $-q^{3}$ & $125 \mathrm{MN} / \mathrm{m}^{3}$ & 2.39 \\
\hline
\end{tabular}

TABLE 2: Estimated nonlinear coefficients (real parts) and ratios in logarithmic scale between their real and imaginary parts. 


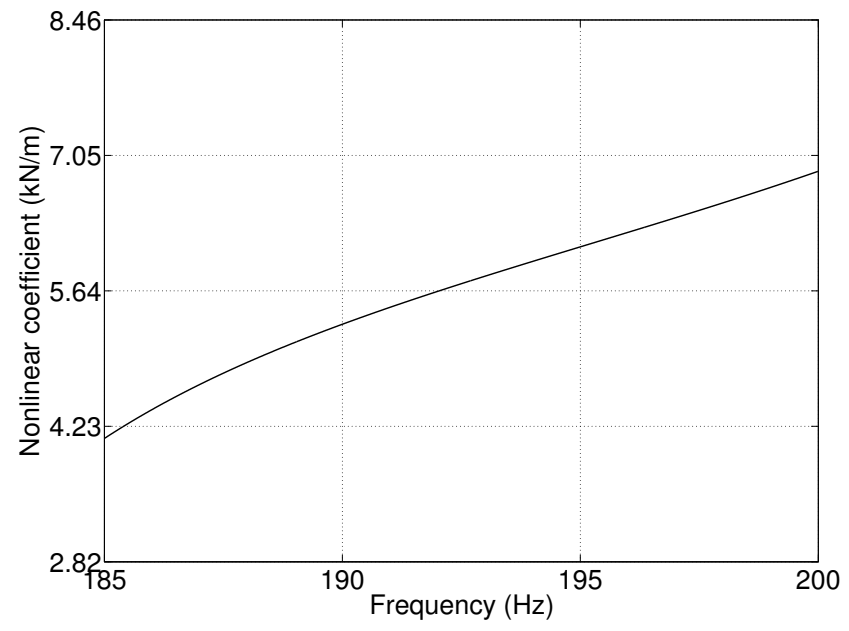

(a)

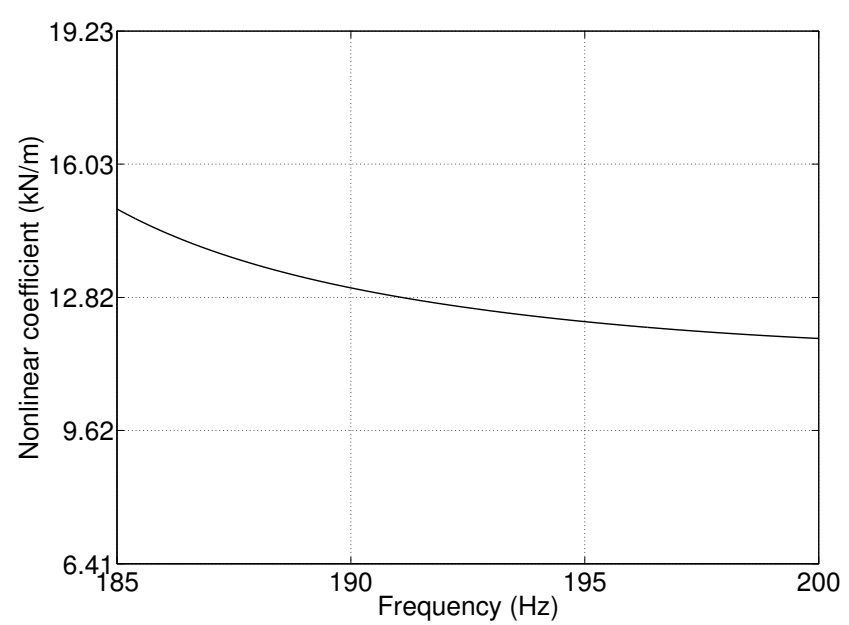

(c)

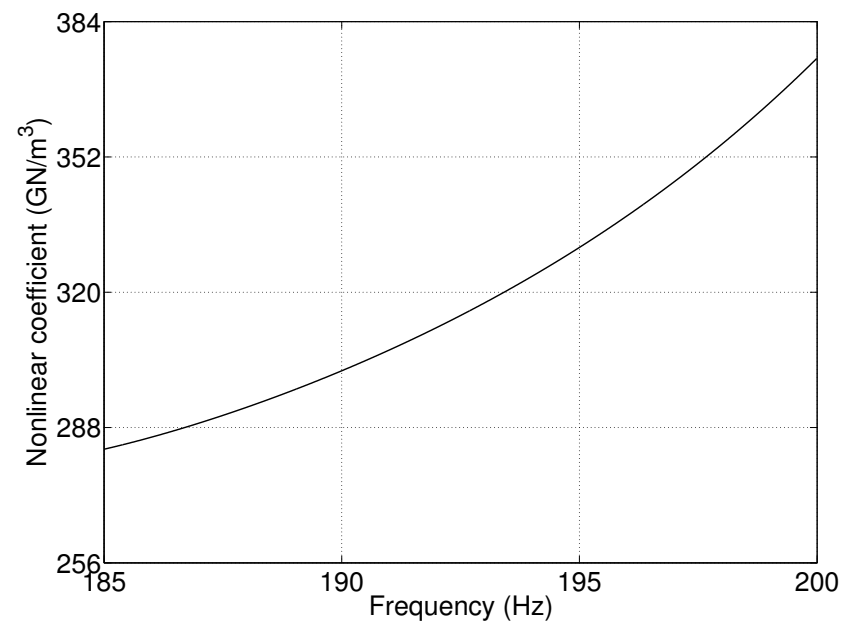

(b)

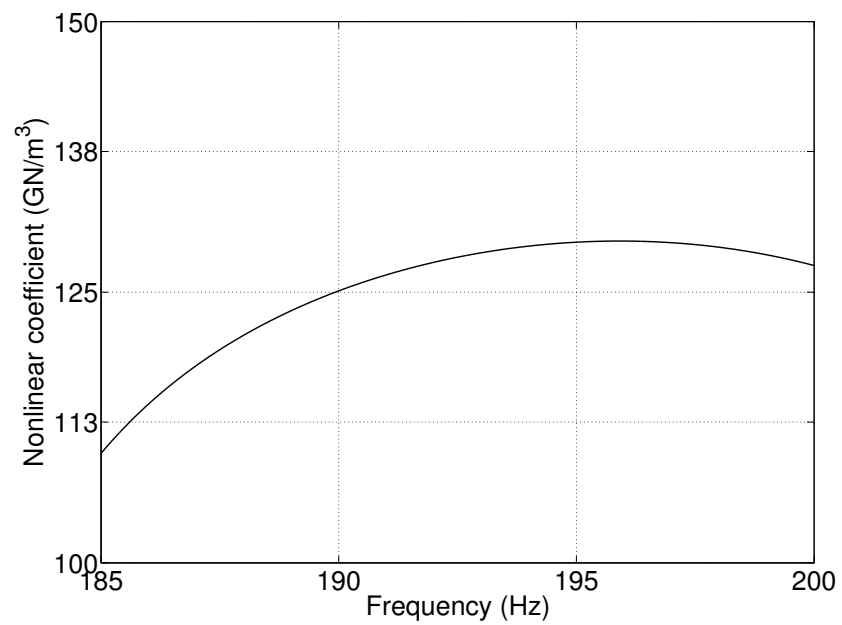

(d)

Figure 9: Real parts of the four nonlinear coefficients estimated by the FNSI method. (a,b) NL 1; (c,d) NL 2. Left column: bilinear coefficients displayed in 50-\% bounds around their mean values; right column: cubic coefficients displayed in $20-\%$ bounds around their mean values. 


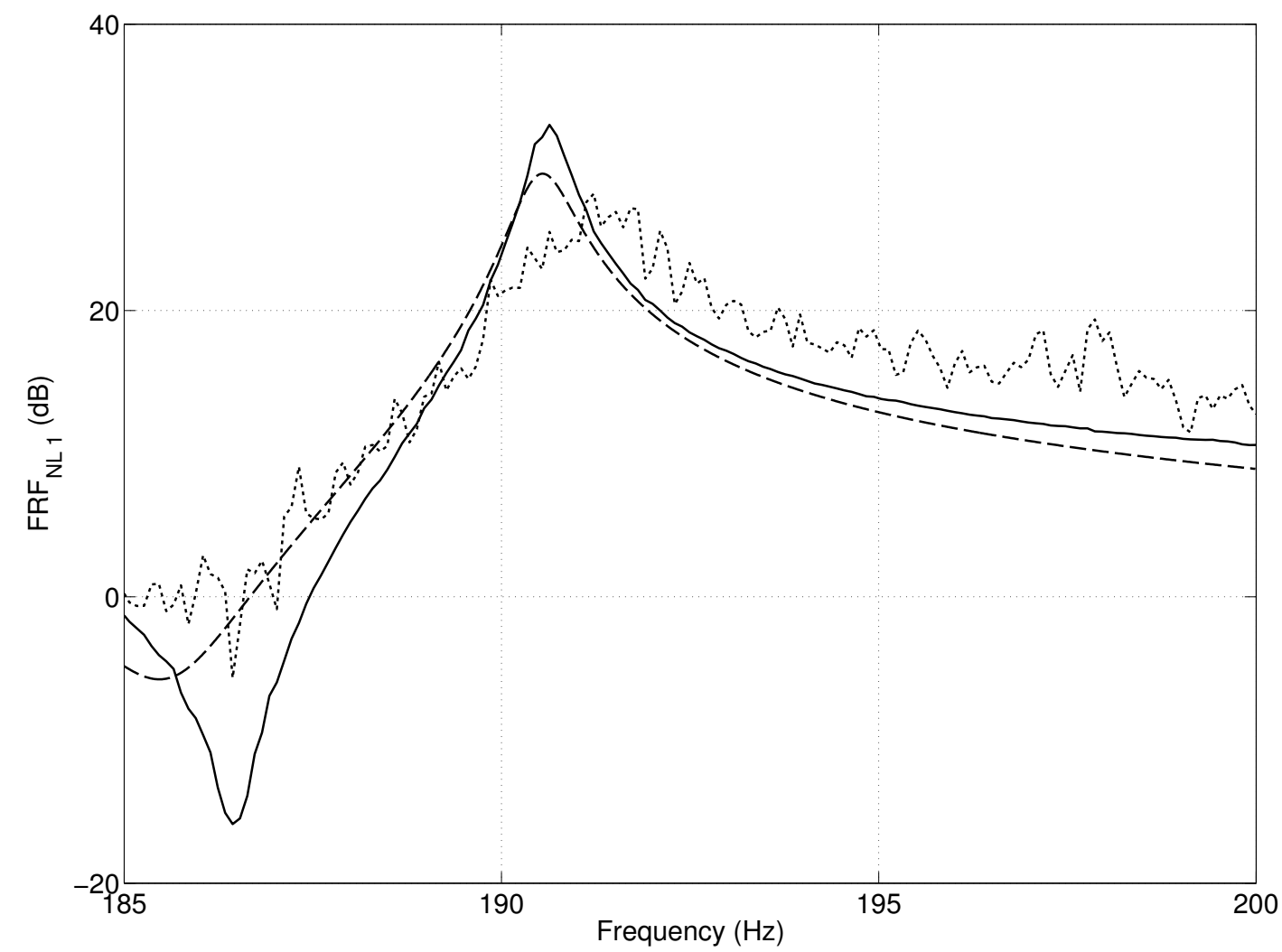

Figure 10: FNSI reconstruction of the FRF in NL 1 from nonlinear data. Solid line: linear estimation at low level (0.16 NRMS); dotted line: linear estimation at high level (3.82 NRMS); dashed line: FNSI estimation at high level (3.82 NRMS). 


\section{REFERENCES}

[1] Van Overschee, P. and De Moor, B., Subspace Identification for Linear Systems, Kluwer Academic Publishers, 1996.

[2] McKelvey, T., Akcay, H. and Ljung, L., Subspace-based multivariable system identification from frequency response data, IEEE Transactions on Automatic Control, Vol. 41, pp. 960-979, 1996.

[3] Reynders, E. and De Roeck, G., Reference-based combined deterministic-stochastic subspace identification for experimental and operational modal analysis, Mechanical Systems and Signal Processing, Vol. 22, pp. 617-637, 2008.

[4] Mevel, L., Hermans, L. and Van Der Auweraer, H., Application of a subspace-based fault detection method to industrial structures, Mechanical Systems and Signal Processing, Vol. 13, pp. 823-838, 1999.

[5] Noël, J. P. and Kerschen, G., A new subspace-based approach to identify nonlinear mechanical structures in the frequency domain, 16th IFAC Symposium on System Identification, 2012.

[6] Noël, J. P., Marchesiello, S. and Kerschen, G., Time- and frequency-domain subspace identification of a nonlinear spacecraft, 25th ISMA International Conference on Noise and Vibration Engineering, 2012.

[7] Kerschen, G., Soula, L., Vergniaud, J. B. and Newerla, A., Assessment of nonlinear system identification methods using the SmallSat spacecraft structure, 29th International Modal Analysis Conference, 2011.

[8] Hot, A., Experimental validation of locally non-linear structures in a space context, Ph.D. thesis, FEMTO-ST Institute, LMARC, 2011.

[9] Kerschen, G., Worden, K., Vakakis, A. F. and Golinval, J. C., Past, present and future of nonlinear system identification in structural dynamics, Mechanical Systems and Signal Processing, Vol. 20, pp. 505-592, 2006.

[10] Adams, D. E. and Allemang, R. J., A frequency domain method for estimating the parameters of a non-linear structural dynamic model through feedback, Mechanical Systems and Signal Processing, Vol. 14, No. 4, pp. 637-656, 2000.

[11] Pintelon, R. and Schoukens, J., System Identification: A Frequency Domain Approach, Wiley-IEEE Press, 2001.

[12] Van Overschee, P. and De Moor, B., Continuous-time frequency domain subspace system identification, Signal Processing, Vol. 52, pp. 179-194, 1996.

[13] Marchesiello, S. and Garibaldi, L., A time domain approach for identifying nonlinear vibrating structures by subspace methods, Mechanical Systems and Signal Processing, Vol. 22, pp. 81-101, 2008. 